\title{
Moyamoya Disease: A Rare Entity Report of One Case
}

\author{
A RAHMAN ${ }^{\mathrm{a}}$, M HAQUE ${ }^{\mathrm{b}}$, MA HOSSAIN ${ }^{\mathrm{c}}$
}

Summary:

Moyamoya disease is a disease of the cerebral vessels at the base of the cranium and is most prevalent in Japan. It is relatively rare in other parts of the world. Though few cases have been reported from different Asian and some European countries, to the best of our knowledge, no case of Moyamoya disease has been reported in our country so far.

\section{Introduction:}

Moyamoya disease is a clinical entity with bilateral stenosis or occlusion of the large intracranial vessels at the base of the brain, with proliferation of fine collaterals. ${ }^{1}$ The term "moyamoya" was introduced by Suzuki and Takaku in 1969 to characterize the angiographic appearance of the condition, which shows a collateral network of blood vessels at the base of the brain. As is described in the text, the Japanese word 'moyamoya' means “something hazy just like a puff of cigarette smoke drifting in the air."2-4

\section{Case Report:}

A 9 years old girl came to us with the complaints of occasional seizures especially following crying or eating hot spicy food. Clinical examination revealed that she is a quite intelligent and playful girl without any neurological deficit. She did not give any history of head injury or family history of epilepsy. Her CBC and other relevant biochemical parameters including blood glucose, renal functions, liver functions and serum electrolytes were within normal limits. So, to

a. Dr. Asifur Rahman; MS

b. Dr. Moududul Haque; Ph D

c. Prof. M Afzal Hossain; FCPS

Department of Neurosurgery, Bangabandhu Sheikh Mujib Medical University, Shahbag, Dhaka-1000, Bangladesh

Address of Correspondence: Dr. Asifur Rahman, Resident. Department of Neurosurgery, Bangabandhu Sheikh Mujib Medical University, Shahbag, Dhaka-1000, Bangladesh

Received: 2 December, 2008

Accepted: 20 December, 2011
Here we report a case of Moyamoya disease in a 9 years old girl diagnosed in the department of neurosurgery of Bangabandhu Sheikh Mujib Medical University. We believe this is the first detected case in our country.

key words: Moyamoya, Puff of smoke, MRI, MR angiogram.

(J Bangladesh Coll Phys Surg 2012; 30: 41-43)

find out the cause of the seizure an MRI of brain was done and in T2WI ( Fig-1 ) and FLAIR ( Fig-2) it showed multiple flow voids in the area of circle of Willis and in the basal ganglia. MR angiogram revealed characteristic Moyamoya vessels with typical "puff of smoke” appearance ( Fig-3 ).

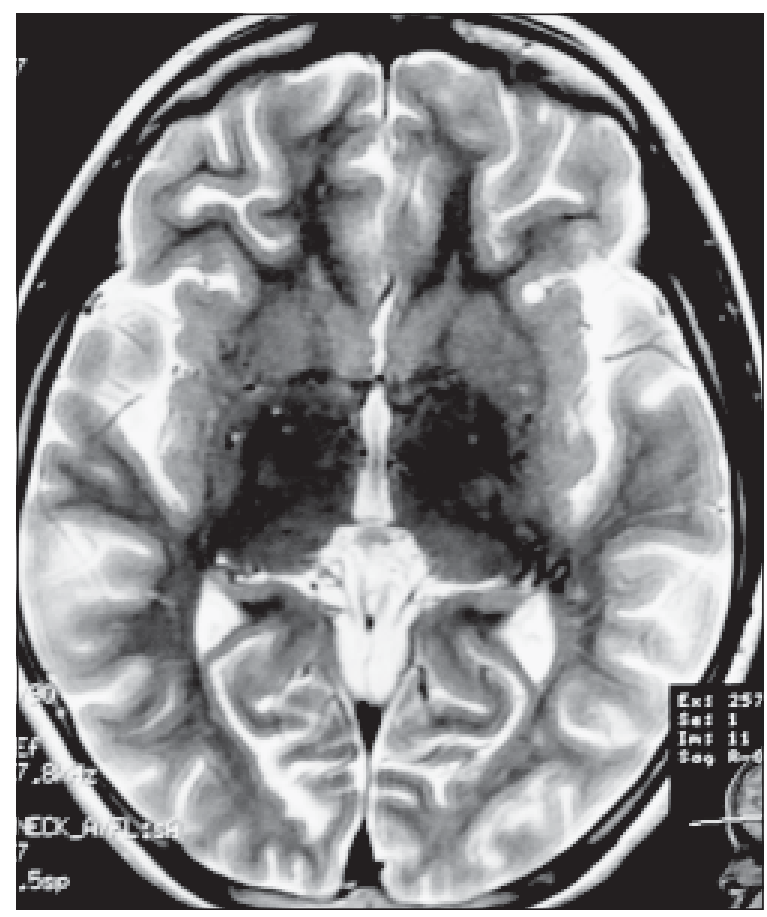

Fig.-1: MRI in T2WI (Axial view) shows multiple flow voids in the area of circle of Willis and in the basal ganglia. 


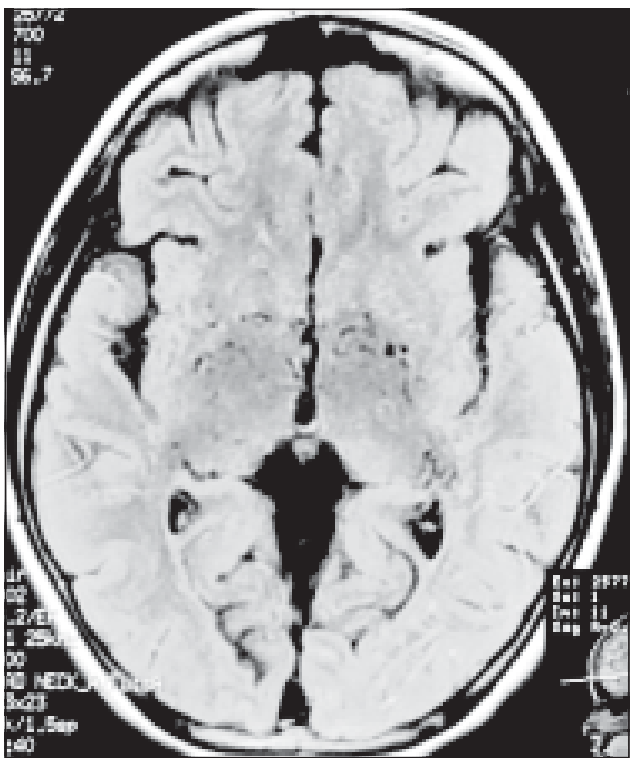

Fig.-2: FLAIR image of MRI (Axial view) showing multiple flow voids in the area of circle of Willis and in the basal ganglia.

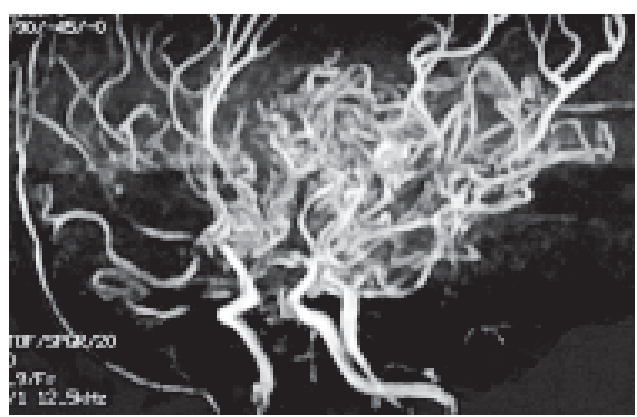

Fig.-3: MR Angiogram ( Right oblique view) showing characteristic Moyamoya vessels with typical "puff of smoke" appearance.

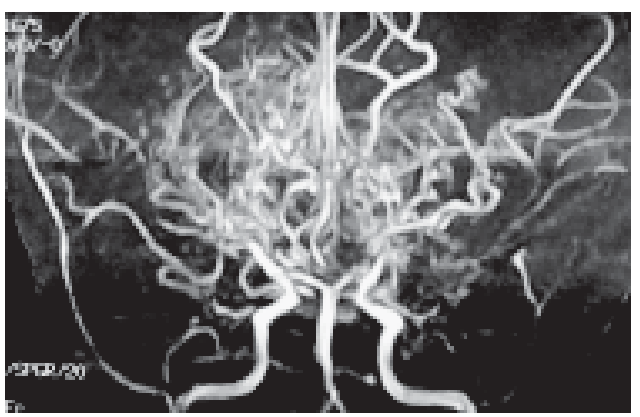

Fig.-4: MR Angiogram (A P view) shows stenosis of bilateral internal carotid arteries.

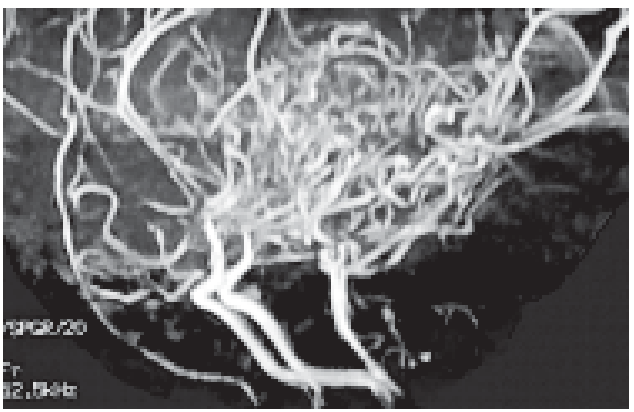

Fig.-5: MR Angiogram (Lateral view) showing collateral Moyamoya vessels with "puff of smoke" appearance.

\section{Discussion:}

Moyamoya disease is a rare idiopathic vaso-occlusive disease characterized by progressive irreversible occlusion of main blood vessels to the brain as they enter into the skull. The blockage tends to cause strokes or seizures. ${ }^{4}$ Many clinical features specific to moyamoya disease have been reported and cited in textbooks. The types of clinical findings can be divided into four subgroups: (a) ischaemia, including infarction and transient ischaemic attacks; (b) haemorrhage; (c) no symptoms and (d) other symptoms, including headaches, seizures and involuntary movements. ${ }^{5}$ Cerebral infarction and disturbed cerebral haemodynamics were detected in $20 \%$ and $40 \%$ of the involved hemispheres, respectively. [06] Our patient presented only with having seizures while under stress and intake of spicy food, most likely because of failure to meet the required metabolic demands from disturbed cerebral haemodynamics. She was otherwise asymptomatic probably because she presented at a very early stage before developing any clinical manifestation other than seizure. A Japanese study also revealed that patients with asymptomatic moyamoya disease are not always rare and the percentage of asymptomatic cases was $17.8 \%$ in Japan. Higher figures than previous reports probably reflect the availability of appropriate diagnostic tools and the brain check-up system that has been extensively developed in Japan. [05] She presented in her first decade and one of the well-known specific features of moyamoya disease is its two-peak pattern of age distribution and its higher incidence in childhood. The first peak being in the first decade and the second being in the fourth decade and the female to male ratio is $2.2: 1 .^{5}$ Though conventional angiography or DSA 
are the investigations of choice, MR angiography has become a reliable diagnostic modality for moyamoya disease. [07] MRI not only reveals areas of infarctions but also allows direct visualization of collateral vessels as multiple small flow voids. MR angiography is used to confirm the diagnosis and to see the anatomy of the vessels involved. It typically reveals the narrowing and occlusion of proximal cerebral vessels and extensive collateral flow through the perforating vessels demonstrating the classic 'puff of smoke' appearance. ${ }^{4}$ The T2WI of MRI of our patient showed multiple flow voids at and around the base of the brain. Her MR angiogram revealed stenosis of the bilateral carotid systems ( Fig-4 ) as well as the typical "puff of smoke" appearance ( Fig-5 ) of collateral vessels. Several surgical procedures, which can be classified as direct and indirect bypass methods, have been proposed for the treatment of this disease. The direct bypass techniques that have been proposed are vein grafts and EC-IC arterial anastomoses (STA-MCA and OA-MCA anastomoses).The indirect techniques are as follows: 1) Encephaloduroarteriosynangiosis; 2) Encephalomyosynangiosis; 3) Encephalomyoarteriosynangiosis; 4) the use of multiple cranial bur holes; and 5) transplantation of omentum. Other options such as cervical carotid sympathectomy and superior cervical perivascular ganglionectomy have also been proposed. Medical management had not been proven capable of controlling or improving the disease. ${ }^{2}$ We explained and counseled the parents about the young girl in details specially regarding the prognosis and outcome of the disease with and/or without surgery and offered for an
EC-IC bypass. The parents informed us that they would let us know their decision later. So, for the time being the girl has been given prophylactic anticonvulsant and is kept under observation at the moment.

\section{Conclusion:}

The patient fulfilled the clinical and angiographic criteria for definitive diagnosis of moyamoya disease, which is a rare entity in our country. And to our knowledge it is the first diagnosed case in Bangladesh.

\section{Reference:}

1. Raghavendra S, Chemmanam T, Kesavadas C, Sarada C. Moyamoya disease. Ann Indian Acad Neurol. 2006;9:124-5.

02. Reis CVC, Safavi-Abbasi S, Zabramski J M., Gusmão SNS, Spetzler RF, Preul MC. The History of Neurosurgical Procedures for Moyamoya Disease. Neurosurg Focus. 2006;20(6).

03. Chang S D. and Steinberg G K. Surgical Management of Moyamoya Disease [homepage on the Internet]. Stanford, California, MoyaMoya.com. [updated 2008 Aug 24; cited 2008 Oct 22]. Available from: http://www.moyamoya.com/ journals/moyamoya.html.

04. Jain AS, Goswami VG, Joshi MA, Patel JM, Shah A, Gupta A. Images : Moya moya disease. Indian J Radiol Imaging. 2004;14:427-9.

05. Baba T, Houkin K and Kuroda S. Novel epidemiological features of moyamoya disease . Journal of Neurology, Neurosurgery, and Psychiatry 2008;79:900-904

06. Kuroda S, Hashimoto N, Yoshimoto T, Iwasaki Y. Radiological Findings, Clinical Course, and Outcome in Asymptomatic Moyamoya Disease, Stroke. 2007;38:1430.

07. Houkin K, Kamiyama H, Abe H, Takahashi A, Kuroda S. Surgical Therapy for Adult Moyamoya Disease. Stroke. 1996; 27:1342-1346. 